\title{
ULTRAVIOLET LASER PHOTOABLATION OF POLYMERS: A REVIEW AND RECENT RESULTS
}

\author{
SYLVAIN LAZARE and VINCENT GRANIER \\ Laboratoire de Photophysique et Photochimie Moléculaire, \\ UA CNRS 348, Université de Bordeaux I, \\ 351 Cours de la Libération, F-33405 Talence, France
}

(Received 17 March, 1988; in final form 6 June, 1988)

\begin{abstract}
The evolution since 1982, of far-UV laser photoablation of polymers is described. The experimental data can be fitted by using a dynamic model which states that the irradiated interface moves at a rate proportional to the difference between, the intensity reaching it, and the ablation threshold intensity $I_{t}$. The screening effect of the ablated gaseous products is taken into account. The experimental etch depth versus fluence, obtained with our new quartz crystal microbalance technique, can be fitted by adjusting two parameters of this model; the mean absorption coefficient of the products $\beta$ and the so-called ablation rate constant $k$, which is the etch rate for $I=I_{t}+1 \mathrm{MW} / \mathrm{cm}^{2}$. These two parameters are wavelength dependent. The model allows also the calculation of the dose of absorbed energy as a function of depth in the material. For each layer of the ablated depth, the dose absorbed before and after (reabsorption of the gas products) ablation are distinguished. The dose of reexcitation varies highly with fluence and the distribution of gas products broadens accordingly. However, primary products need to be studied. Surface products are formed on the final surface, during the postablation phase of the pulsed irradiation. The probable mechanism of the evolution of excitation energy is discussed, with stress on the elementary steps leading to heat production.

The perspectives of evolution of etching and patterning polymer surfaces by far-UV photoablation are high in basic research and technology.
\end{abstract}

KEY WORDS: Photoablation, far-UV lasers, polymer, surface, dynamic model, etch rate, mechanism, application.

\section{INTRODUCTION}

In the past six years, ${ }^{1}$ photoablation of materials with the high intensity UV light of lasers has gained in popularity in many development and basic research laboratories. This may be attributed to the recent progress made in the laser field and in particular to the development of excimer lasers, ${ }^{2}$ which were born ten years ago. ${ }^{3}$

Photoablation ${ }^{4}$ consists in the spontaneous etching that occurs upon the absorption, at the material surface, of a pulse of laser light, whose energy is greater than the ablation threshold value. The advantage of using UV light resides in the fact that the ablation is strictly confined to the volume that absorbs the energy. Owing to the high absorption coefficient in far-UV of many materials this volume is very small (of the order of a fraction of a micron in depth). In other words, the laser energy is, for most materials very densely absorbed at the surface. The ablative conditions are rapidly reached during the excitation pulse and the volatile products eventually formed are 
quickly propelled outside the solid. Additionally, thanks to low thresholds of ablation, focussing of the laser beam is not necessary, and large surface areas can be treated. A number of materials can be patterned by this technique, with a submicronic resolution..$^{5}$ Applications of photoablation are already developed in many fields like for instance, microlithography, ${ }^{6}$ microsurgery ${ }^{7}$ (keratoplasty, ${ }^{8}$ angioplasty ${ }^{9}$ and neurosurgery $\left.{ }^{10}\right)$, micromachining of materials, ${ }^{11}$ vaporization of refractory solids ${ }^{12}$ (for the synthesis of new substances or thin films), and characterization of surfaces ${ }^{13}$ (LAMMA). Future applications are envisaged like the determination of the depth profile of properties in materials. All these applications require a better understanding of the interaction of the pulsed high intensity radiation $\left(>1 \mathrm{MW} / \mathrm{cm}^{2}\right)$ with the solid and this is the aim of the present basic research on photoablation.

Polymers, in general, have a strong sensitivity to UV laser photoablative conditions. They have ablation thresholds $\left(10-20 \mathrm{~mJ} / \mathrm{cm}^{2}\right)$ lower than that of metals ${ }^{14}$ $\left(4 \mathrm{~J} / \mathrm{cm}^{2}\right.$ at $248 \mathrm{~nm}$ for copper) semiconductors ${ }^{15}\left(1.4 \mathrm{~J} / \mathrm{cm}^{2}\right.$ at both 193 and $248 \mathrm{~nm}$ for silicon) and metal oxides superconductors ${ }^{16}\left(110 \mathrm{~mJ} / \mathrm{cm}^{2}\right.$ at $248 \mathrm{~nm}$ for $\mathrm{YBaCuO}$ ). They also have a much higher rate of ablation, which can be measured as the ablated depth per pulse. ${ }^{17}$

Biological tissue ${ }^{18}$ can also be etched or cut beautifully with a high lateral and depth resolution by UV-laser photoablation. These peculiar materials are constituted either of extracellular proteinic macromolecules or living cells and can be viewed as polymeric gels, or polymer swollen with a large amount of water. The presence of water, which is essentially non-absorbing in UV does not affect significantly the course nor the cleanliness of the photoablative cut. Nevertheless, the threshold of ablation is increased by a dilution effect ${ }^{19}\left(100\right.$ and $500 \mathrm{~mJ} / \mathrm{cm}^{2}$ at 193 and $248 \mathrm{~nm}$ respectively for the cornea tissue). The mechanism of ablation of these materials is expected to be somewhat different from that of the ablation of plain polymers.

Both synthetic polymers and biological materials are very sensitive to heat. In many cases it was seen that UV-laser photoablation does not damage these substrates. ${ }^{20} \mathrm{~A}$ chemical modification ${ }^{21}$ occurs at the etched surface in a film whose thickness is limited to a few thousands of angstroms or less. ${ }^{22}$ Sometimes, melting of the surface, taking place during the laser pulse, is seen over a fraction of a micron. When the polymer is swollen with small molecules, the fast evaporation of the solvent efficiently cools the excited interface.

The present main concern of basic studies is to validate a detailed mechanism. The time dependence of photoablation was predicted to be in the picosecond range by the theoretical model of Garrison and Srinivasan ${ }^{23}$ in 1985 and was confirmed experimentally ${ }^{24}$ in 1986. For the nanosecond pulses of the excimer laser, the process can be viewed as occurring continuously during the absorption of the excitation pulse and not like an explosion taking place after the absorption of the radiation. Therefore, as suggested by Keyes et al. ${ }^{25}$ the interface is moving during the irradiation. The use of picosecond and femtosecond pulses may lead to ablation with a different time profile. In fact, the experiments have shown that the instantaneous intensity only is important. And this is also in agreement with the dynamic model developed by Sutcliffe and Srinivasan. ${ }^{26}$ 


\section{TIME DEPENDENT MODEL OF PHOTOABLATION}

It is now clear that laser photoablation with far-UV $(180-300 \mathrm{~nm})$ is a dynamic phenomenon due to the high intensity of the pulsed radiation. It is triggered when the peak intensity $I$ reaches the ablation threshold (of the order of $1-2 \mathrm{MW} / \mathrm{cm}^{2}$ for highly absorbing polymers). The irradiated interface moves as long as the intensity that is falling on it, is exceeding $I_{t}$. Figure 1 shows an instantaneous picture of the ablation phase and, in Figure 2, are defined the three phases ( $\Phi \mathrm{I}, \Phi \mathrm{II}, \Phi \mathrm{III})$ of the phenomenon: preablation, ablation and postablation. During $\Phi I$ and $\Phi I I I$, the substrate surface does not move, but it receives some energy at an intensity lower than the ablation threshold. During $\Phi I I$, the moving interface must see a radiation intensity $I(t)=I_{0}(t) e^{-\beta(t) x(t)}$, which takes into account the screening effect ${ }^{27}$ of the expelled products whose absorption coefficient is $\beta(t)$. $I_{0}(t)$ is the intensity profile of the pulse, and $x(t)$ is the position of the interface at time $t$, referenced to the initial position. During ablation this intensity is above $I_{t}$ and the rest of the incident laser energy must be absorbed by the expelled fragments $I_{0}(t)\left(1-e^{-\beta(t) x t}\right)$ (screening effect). As indicated in Figure 2 the instantaneous speed $v$ can be assumed to be proportional to the difference between the laser intensity reaching the surface and the threshold of ablation:

$$
v=k\left(I_{0} e^{-\beta x}-I_{t}\right) .
$$

Here $v$ is expressed in $\AA / n s e c, \beta(t)$ is the dynamic absorption coefficient of the expanding gas phase (averaged over the gas phase thickness), $x(t)$ is the position of the interface at time $t$ and $k$ is the rate constant expressed conveniently in $\AA \cdot n \sec ^{-1}$.

\section{PHOTOABLATION}

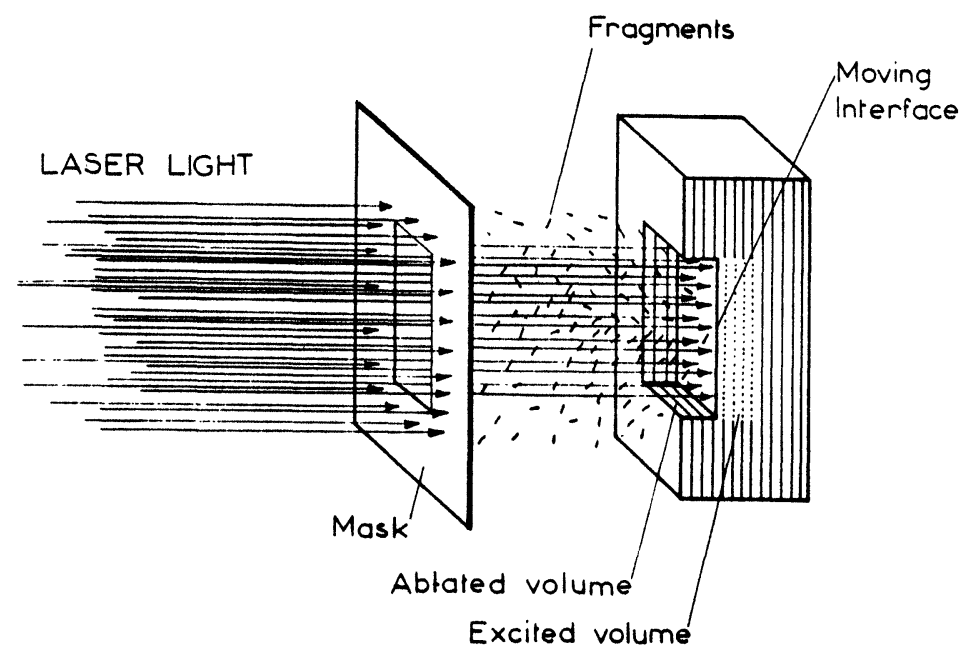

Figure 1 Instantaneous picture of photoablation with the far-UV radiation of the excimer laser, based on the model of the moving interface, whose rate is proportional to $I_{0}(t) e^{-\beta x}-I_{t}$. 


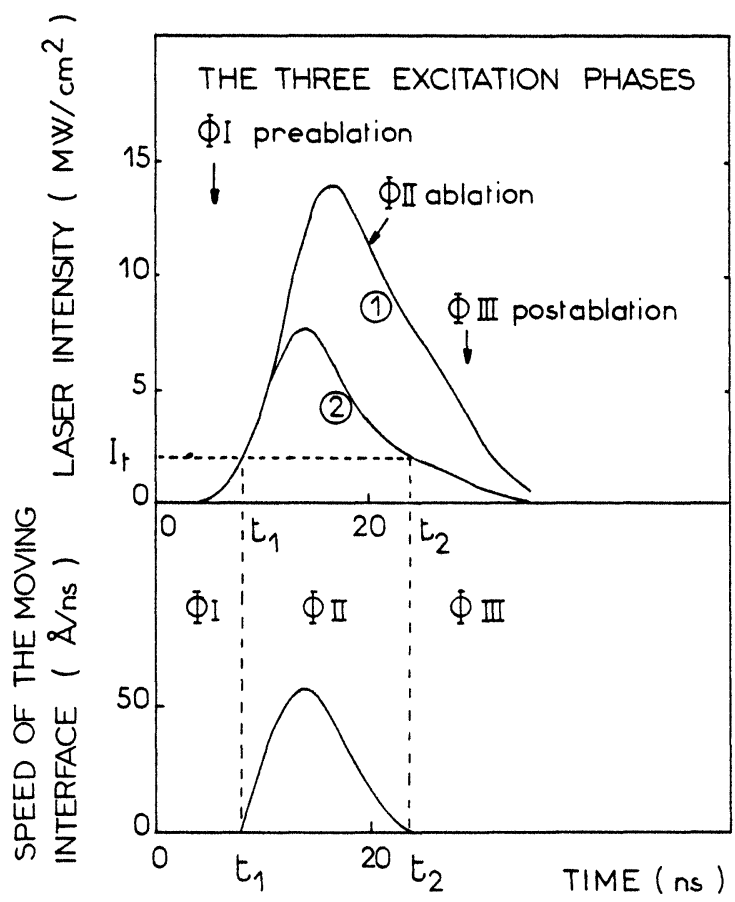

Figure 2 Time profile of the intensity (curve 1) of an excimer laser pulse $\left(193 \mathrm{~nm}, 200 \mathrm{~mJ} / \mathrm{cm}^{2}\right)$ with the three excitation phases of the ablation process; preablation, ablation and postablation. Ablation occurs . when the intensity reaching the surface (curve 2, from the model applied to PET), after absorption by the gas products, is larger than the threshold $I_{t}$. The lower window shows the instantaneous rate of the moving interface computed from the model fitted to PET, for same laser pulse.

$\mathrm{MW}^{-1} \cdot \mathrm{cm}^{2}$. An important aspect of this model is that the absorption of the light and ablation are simultaneous in $\Phi \mathrm{II}$.

\section{DYNAMIC ABSORPTION}

In $\Phi \mathrm{I}$ absorption only occurs and it obeys a dynamic Lambert-Beer law $I(t)=$ $I_{0}(t) e^{-\alpha(t) x}, x$ being the distance below the surface at which the energy is transmitted and $\alpha(t)$ the dynamic absorption coefficient of the solid. At the high intensity of the laser, the absorption coefficient can be highly modified by the energy already absorbed. Figure 3 shows several expected dynamic behaviour of the absorption coefficient of a solid polymer, under high intensity irradiation. The change can be only transient, as the one due to short lived electronic excited states or radicals, or can be permanent, as the one due to the formation of new chemical structures. As a direct consequence of the dynamic character of the absorption coefficient, those measured at low intensity (from the absorption spectrum) are almost useless in interpreting the dynamic measurables of photoablation, like intensity threshold $I_{t}$ and ablation rate constant $k$. The experiments show that there is absolutely no 


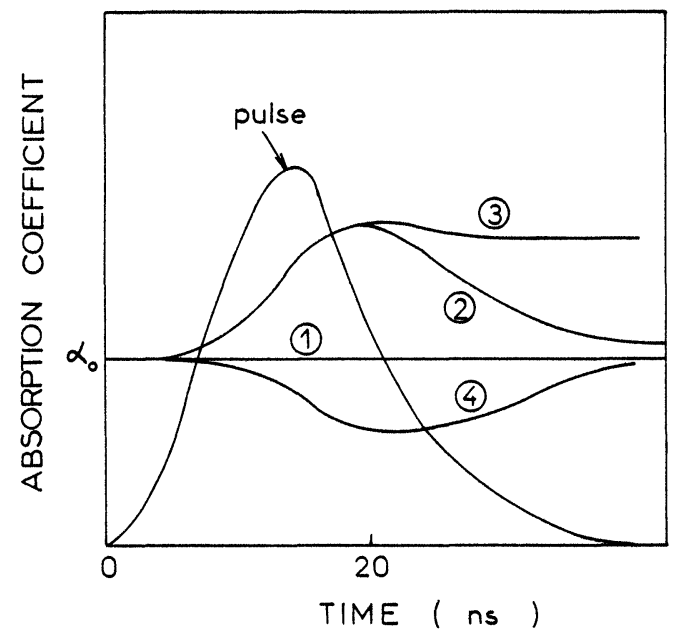

Figure 3 Probable time profile of the absorption coefficient $\alpha$ of the solid polymer under irradiation. (1) $\alpha$ constant; (2) Increase and recovery of the initial value; (3) Transient and permanent increase; transient decrease "bleaching of the material."

correlation between ablation rate or threshold and these coefficients. Attempts to measure the dynamic coefficient of the polymeric substrate are reported by two groups. ${ }^{28,29}$

\section{ABLATION THRESHOLD}

The threshold of ablation (peak intensity threshold) represents the minimum energy per unit of time and volume required to overcome the reformation of the polymer broken bonds and to give to the products enough kinetic energy to escape from the irradiated volume. The volumic threshold is given by

$$
I_{t}\left(t_{1}\right)=\alpha\left(t_{1}\right) I_{0}\left(t_{1}\right)
$$

It is also a dynamic quantity and its value is therefore not predictable from the low intensity coefficient $\alpha(0)$, since $\alpha\left(t_{1}\right)$ depends on the energy already absorbed at time $t_{1}$ :

$$
E_{t}\left(t_{1}\right)=\int_{0}^{t_{1}} \alpha(t) I_{0}(t) d t
$$

We see that the ablation threshold can be reached dynamically by an intensity induced absorption. This explains why some polymers that are poorly absorbing at low intensity have relatively low ablation thresholds. This is the case of poly(methyl methacrylate) which is weakly absorbing at $193 \mathrm{~nm}$ and almost nonabsorbing at $248 \mathrm{~nm}$ and has nevertheless low ablation thresholds as indicated in Table 1. The experimental threshold can be expressed in intensity or fluence since these two numbers are proportional. 
Table 1 Ablation thresholds $F_{t}$ in $\mathrm{mJ} / \mathrm{cm}^{2}$ (fluence threshold) and low intensity absorption coefficients $\alpha$ in $\mathrm{cm}^{-1}$ for various polymers at two wavelengths of the excimer laser

\begin{tabular}{llllllll}
\hline & & $P S$ & $P C$ & $P E T$ & $P I$ & $P M M A$ & $P P Q$ \\
\hline $193 \mathrm{~nm}$ & $\alpha$ & $8 \times 10^{5}$ & $5.5 \times 10^{5}$ & $3 \times 10^{5}$ & $4.2 \times 10^{5}$ & $2 \times 10^{3}$ & $0.28 \times 10^{3}$ \\
& $F_{t}$ & 10 & 16 & 17 & 27 & 27 & 27 \\
$248 \mathrm{~nm}$ & $\alpha$ & $6.3 \times 10^{3}$ & $1 \times 10^{5}$ & $1.6 \times 10^{5}$ & $2.8 \times 10^{5}$ & 65 & $0.16 \times 10^{5}$ \\
& $F_{t}$ & 57 & 56 & 22 & 65 & 200 & 37 \\
\hline
\end{tabular}

PS: polystyrene.

$P C$ : polycarbonate.

PET: poly(ethylene terephthalate).

$P I$ : polyimide.

$P M M A$ : poly(methyl methacrylate)

$P P Q$ : polyphenylquinoxaline.

The experimental threshold is the minimum pulse energy that produces ablation. Of course, this is not independent of the sensitivity of the means that is used to detect the onset of ablation. We have recently demonstrated that the quartz crystal microbalance ${ }^{30,32}$ is the most sensitive technique to record the ablation of minute amount (less than $1 \AA$ per pulse) of material. Much lower values than those obtained with other methods (profilometry) were measured (see Table 1).

Etching of non-absorbing polymers (for instance polyethylene, polypropylene, polytetrafluoroethylene, etc.) or etching by using longer wavelengths $(>300 \mathrm{~nm})$ requires a much higher intensity in order to reach the threshold of absorbed energy. In these cases, the chances of bulk damage, by photochemistry, thermolysis, or dielectric breakdown, are increased. However, the recent development of new femtosecond lasers, has allowed coherent two-photon absorption leading to a clean photoablation $^{31}$ at a wavelength $(308 \mathrm{~nm})$ that is not absorbed by PMMA at low intensity.

\section{PHOTOABLATION RATE}

The aim is to relate the instantaneous rate of ablation to the incident intensity. In the dynamic model introduced above, the speed of the moving interface can be used to characterize the rate of ablation. The integration of this quantity over one pulse gives the measurable etch depth. Profilometry ${ }^{32}$ and Scanning Electron Microscopy ${ }^{33}$ have been used, since 1982, to appreciate the etch depth per pulse. Since they lack precision for measuring low etch rate, we have recently developed a new technique which is based on the use of a quartz crystal microbalance. ${ }^{22}$ It has shown a superior accuracy in measuring the etch depth for the polymers listed in Scheme 1 at two wavelengths of the excimer laser (193 and $248 \mathrm{~nm}$ ). The curves giving the etch depth as a function of fluence for poly (ethylene terephthalate) are displayed in Figure 4 and Figure 5 for $193 \mathrm{~nm}$ and $248 \mathrm{~nm}$ respectively. They are very similar in shape, but 248 $\mathrm{nm}$ etches the polymer faster. Up to approximately one Joule $/ \mathrm{cm}^{2}$, they both can be fitted by the dynamic model of the moving interface defined above, with the aid of some approximations given below. The etched depth is given by integrating the instantaneous speed of the interface on the pulse duration and taking an intensity 
PS : polystyrene<smiles>CCc1ccccc1C</smiles>

PC. polycarbonale<smiles>COc1ccc(C(C)(C)c2ccc(OC(C)=O)cc2)cc1</smiles>

$P P Q$ : polyphenyl quinoxaline<smiles></smiles>

PET : poly(ethylene terephthalate)<smiles>COCCCOC(=O)c1ccc(C(C)=O)cc1</smiles>

\section{Scheme 1}

profile as given in Figure 2

$$
d(F)=\int_{t_{1}}^{t_{2}} v(t) d t, \quad \text { where } F \text { is the fluence. }
$$

The pulse intensity profile (taken from Ref. 26) is identical at $193 \mathrm{~nm}$ and $248 \mathrm{~nm}$ and approximations were taken as follows. A possible reflection of the laser radiation at the polymer surface is neglected. $\beta, k$ and $I_{t}$ are considered as constant. A good fit

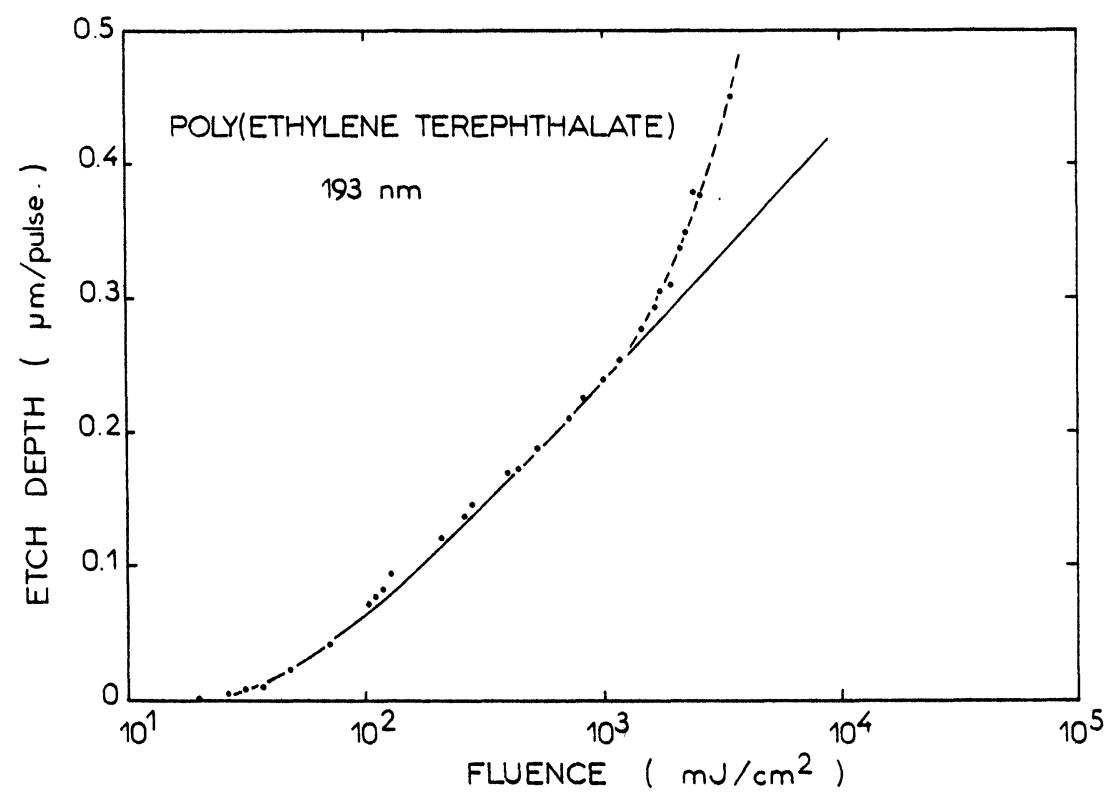

Figure 4 PET etch depth per pulse vs log fluence of the $193 \mathrm{~nm}$ radiation. The solid line is the computed curve by using the model of the moving interface. 


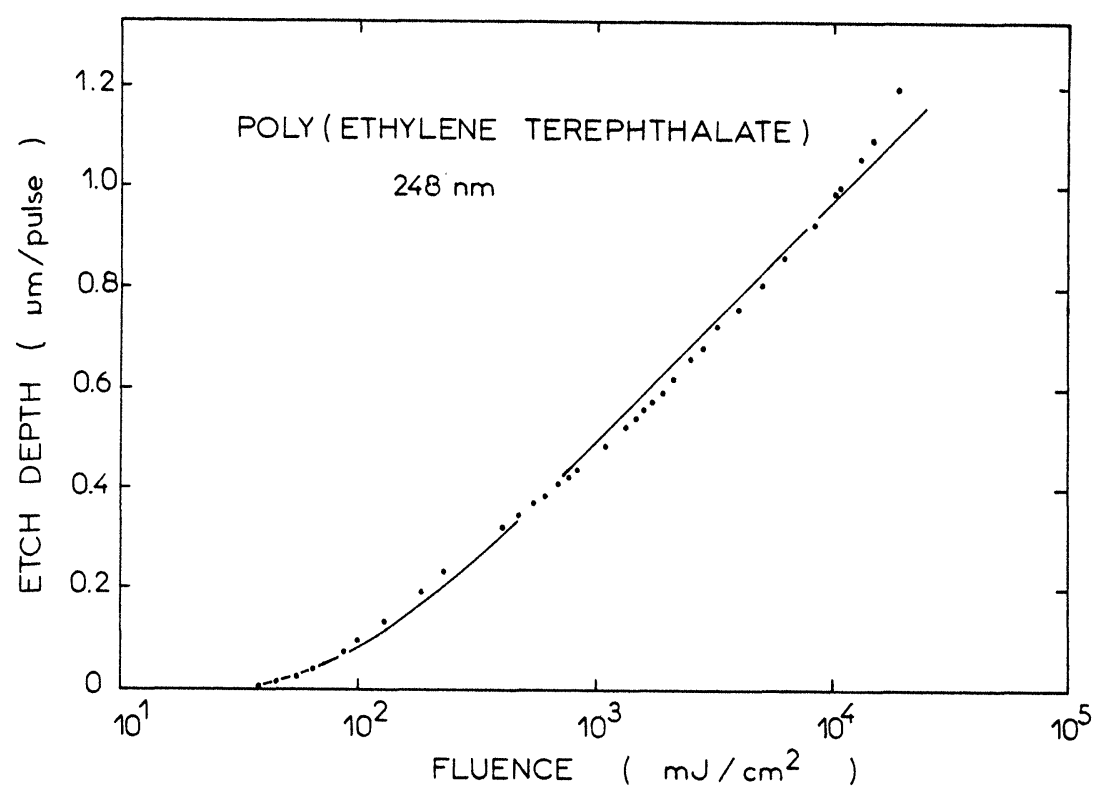

Figure 5 PET etch depth per pulse vs log fluence of the $248 \mathrm{~nm}$ radiation. The solid line is the computed curve by using the model of the moving interface.

gives an averaged value of $\beta$, absorption coefficient of the gas phase products, and $k$ the ablation rate constant. $\beta$ quantifies the reabsorption of the upcoming part of the pulse by the ablated fragments during their flight in the laser beam. The constant $k$, defined as the rate constant, represents the rate of ablation for an incident intensity of $1 \mathrm{MW} / \mathrm{cm}^{2}$ over the threshold value. The threshold of ablation is determined experimentally and the fit gives a unique couple of values for $\beta$ and $k$. These parameters, for poly(ethylene terephthalate) are displayed in Table 2 for two different wavelengths. Similar fits have been obtained for other materials and the parameter $k$ will be exploited to compare different polymers and to compute the averaged dynamic absorption coefficient $\alpha(t)$ of the solid polymer. However one of these fits, which are excellent, is not completely satisfactory at the high fluence $\left(>1 \mathrm{~J} / \mathrm{cm}^{2}\right)$ of the $193 \mathrm{~nm}$ radiation, which is the most densely absorbed by either the gas fragments or the starting polymer. This probably denotes the apparition of the contribution of a new ablation mechanism and is confirmed by the curve of the degraded depth displayed in Figure 7 showing a dramatic increase (see comments below) in this range of fluence. In particular we may think of a plasma mechanism as previously put

Table 2 Ablation rate constant and averaged coefficient of the reabsorption of the expanding fragments of ablation

\begin{tabular}{lll}
\hline PET & $193 \mathrm{~nm}$ & $248 \mathrm{~nm}$ \\
\hline$\beta \mathrm{cm}^{-1}$ & $1.2 \times 10^{5}$ & $0.5 \times 10^{5}$ \\
$k \AA \cdot \mathrm{ns}^{-1} \cdot \mathrm{MW}^{-1} \cdot \mathrm{cm}^{2}$ & 22 & 25 \\
\hline
\end{tabular}


forward by several authors ${ }^{34}$ in the case of ablation of semiconductors or metals. This hypothesis, however needs further investigation.

\section{PHOTOLYSIS PRODUCTS}

There are two types of products. Gas products that are expelled from the solid during ablation and solid products that remain on the new surface and are formed mainly during the postablation phase; stable products that can be isolated by condensing the gas phase ${ }^{35}$ and analyzed, are not primary products of ablation since they have undergone many collisions and reexcitations; unstable transient products are specially formed in the gas phase and can be seen by fast spectroscopy techniques, like fluorescence ${ }^{36}$ and laser induced fluorescence ${ }^{37}$ or can be detected when the ablation is, performed in a mass spectrometer. ${ }^{38}$ They are constituted of a complex mixture of fragments ranging from single atoms to molecules of larger size (solid particles have also been seen). Since they are expelled in the laser beam, they reabsorb many photons before the end of the pulse, and are consequently transformed. Figure 6 gives the proportion of energy absorbed by one single layer before and after ablation. Since the high intensity absorption coefficient of PET at $193 \mathrm{~nm}$ is not available, the calculation was done by using the low intensity one, which is high and therefore presumably relatively independent on time or intensity. We see that even at moderate fluence $\left(100 \mathrm{~mJ} / \mathrm{cm}^{2}\right.$, for PET) the primary products of ablation reabsorb a considerable amount of incident energy. It seems unlikely that they

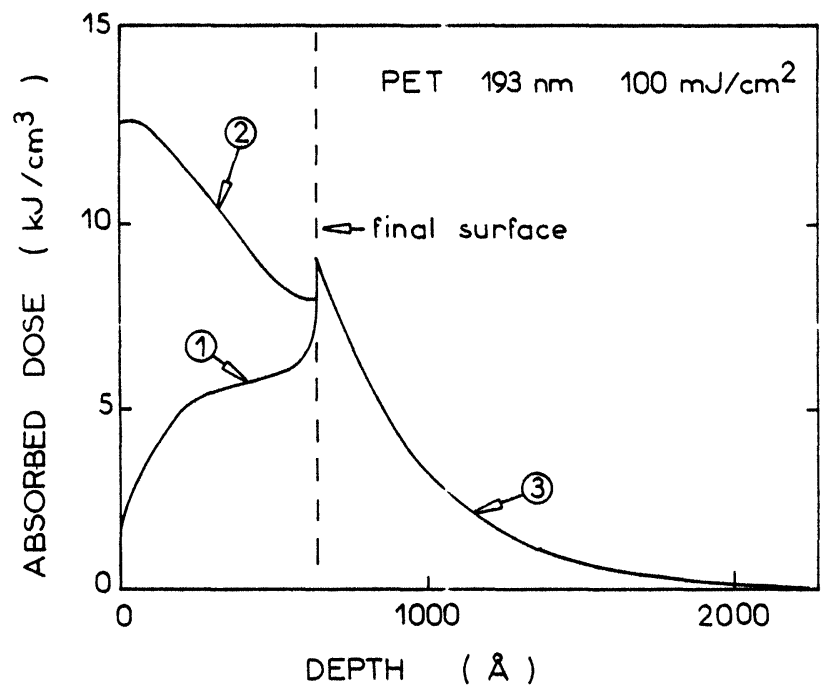

Figure 6 Profile of the deposited energy, as a function of depth into a PET sample, integrated over one laser pulse $\left(193 \mathrm{~nm}, 100 \mathrm{~mJ} / \mathrm{cm}^{2}\right)$. Curve 1 is the energy accumulated before ablation. Curve 2 is the total dose accumulated and the difference between 2 and 1 is the energy absorbed by the gaseous products during their flight outside the irradiated volume. Curve 3 is the total energy accumulated under the final interface. These data were obtained by using the model of the moving interface, and for simplicity an absorption coefficient equal to $3 \times 10^{5} \mathrm{~cm}^{-1}$, the low intensity value, was used. 
survive very long to this reexcitation. The curve of Figure 6 is computed by using the dynamic model presented before. We can see from this graph that each ablated layer does not receive the same dose of energy and necessarily does not give the same distribution of products. From this model, we can therefore predict that the identification of the gas products is complex since it depends on many parameters like fluence, depth and, of course, the environment (air or vacuum). Despite this complexity, there is a high interest in products determination for the elucidation of the mechanism, the toxicology in medical applications ${ }^{39}$ and the contamination ${ }^{40}$ by redeposition in the technological applications. By using a fluence close to the ablation threshold, one may be able to collect gas products close enough to primary ablation products, since in this case reabsorption is minimized.

Among the primary products $\mathrm{C}_{2}$ (from PMMA), $\mathrm{C}_{2}$ and $\mathrm{CN}$ (from PI) have been tracked by laser induced fluorescence. ${ }^{37}$ Their velocity distribution is peaking at about $0.8 \mathrm{~cm} / \mu$ s for ablation of PMMA at $248 \mathrm{~nm}$ at a fluence around $100 \mathrm{~mJ} / \mathrm{cm}^{2}$. Upon rising the fluence, this value is expectedly increased. Velocity distribution of larger molecules like methyl methacrylate (from PMMA) or styrene (from PS) were studied by time of flight (TOF) mass spectrometry or picosecond laser ionization TOF mass spectrometry. ${ }^{38}$ The results agree to demonstrate that the velocity distribution is narrower than that of a Maxwell-Boltzman distribution of a high temperature thermal equilibrium. In general, larger fragments are expelled at lower speed. This high average speed (appr. $1000 \mathrm{~m} / \mathrm{s}$ ) of the gas products distribution is acquired during the pulse duration. The etched substrate therefore undergoes a transient stress that has been measured ${ }^{24}$ and used to appreciate the time profile of the ablation phenomenon. The polymer to ablate was put on a fast pressure sensitive detector made of piezoelectric polyvinylidene fluoride. The signal was as short as the excitation pulse. Some recent experiments, ${ }^{41}$ based on a fast refractive index measurement have shown that at moderate fluence $\left(<1 \mathrm{~J} / \mathrm{cm}^{2}\right)$ no free electron is produced in the gas phase indicating that no plasma is generated. However, molecular ions may be present as the result electron transfer occurring during the collisions.

The solid products, remaining on the surface, are formed by excitation with the postablation part of the pulse (phase III in Figure 2). In the case of poly(ethylene terephthalate), ${ }^{21}$ their stoechiometry was studied by X-ray photoelectron spectroscopy (XPS) in different conditions of fluence and wavelength and their solubility was determined by using the quartz crystal microbalance. ${ }^{30}$ They are contained in a thin layer whose thickness is around $1000 \AA$ (related to the penetration of the light) and are composed of a mixture of oligomers. They contain more carbon atoms than the original polymer owing to the formation of inert molecules like $\mathrm{CO}$ and $\mathrm{CO}_{2}$ upon photolysis (Figure 8). From scanning electron microscopy pictures of the surface, it is evident that they are formed in the liquid state. A striking experimental fact is that the formation of these products increases abruptly (as measured by the thickness ${ }^{22}$ ) when the fluence is several times the ablation threshold as seen in Figure 7. This sudden efficiency is attributed to the melting of the polymer surface which, by increasing the molecular diffusion would disfavour the recombinaison. A second unexpected effect is the increase that is seen at much higher fluence $\left(>1 \mathrm{~J} / \mathrm{cm}^{2}\right)$ for 


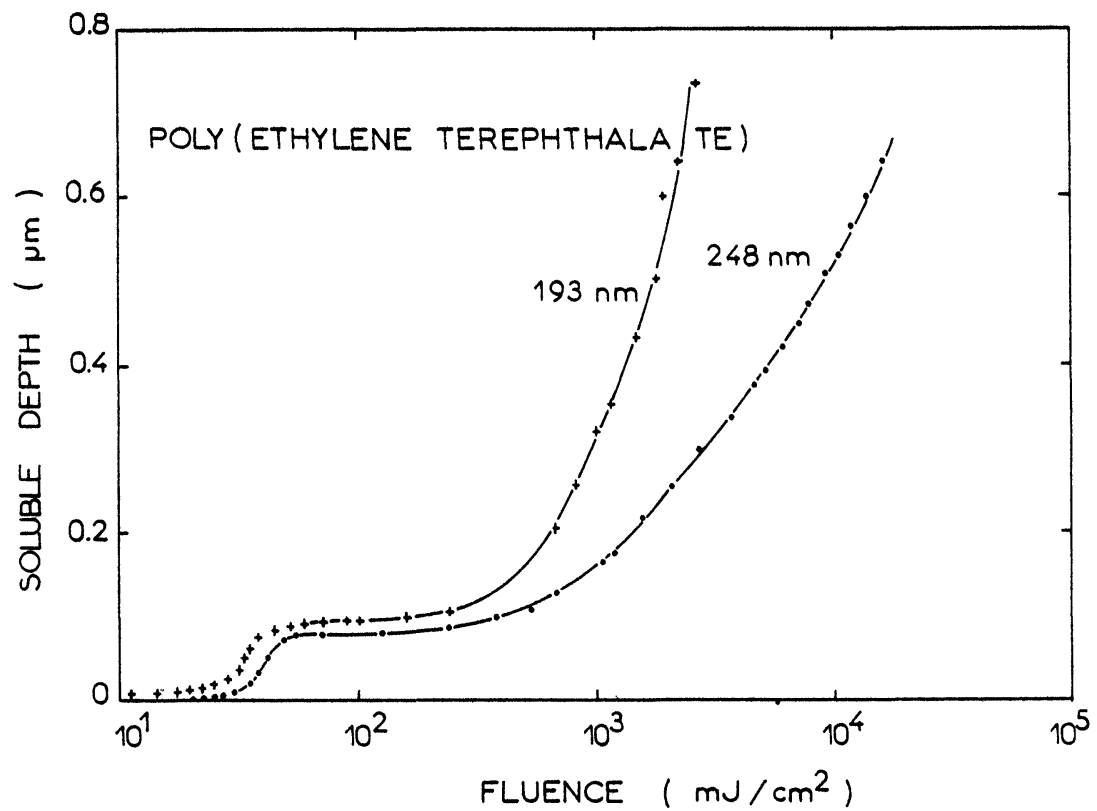

Figure 7 Soluble depth (in acetone) as a function of fluence obtained by far-UV irradiation of PET surface at 193 and $248 \mathrm{~nm}$.

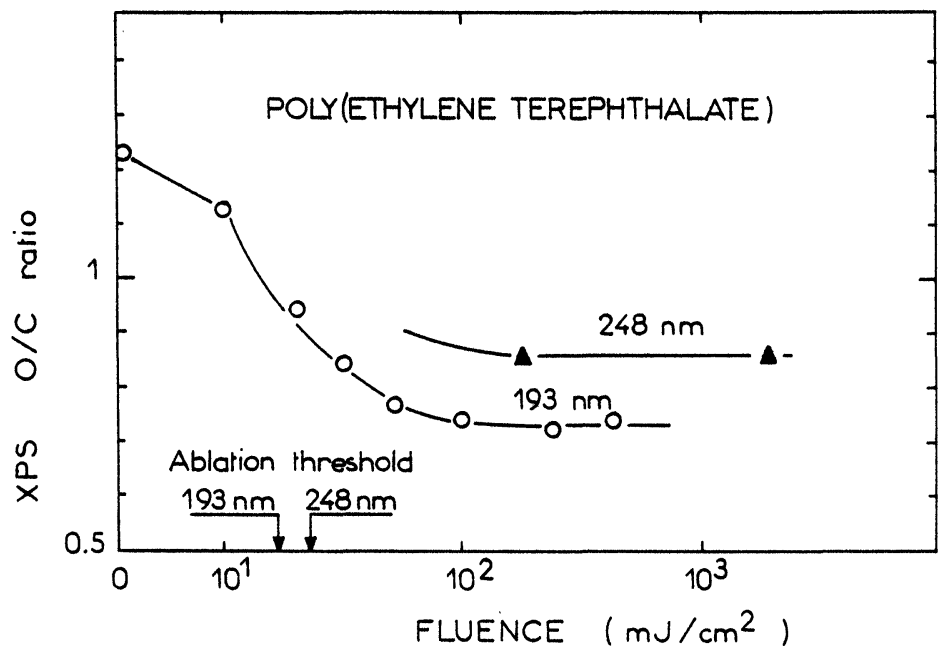

Figure. 8 X-ray photoelectron spectroscopy (XPS) O1s/C1s intensity ratio, as a function of fluence, of PET surface irradiated with the 193 or $248 \mathrm{~nm}$ radiation. The sampling depth of the XPS technique is $50 \AA$. 
both 193 and $248 \mathrm{~nm}$. It cannot be due to an increase of the postablation energy since a high dose low intensity irradiation does not solubilize more than $1000 \AA$ at the surface. This is probably due to the penetration of a radiation of longer wavelength which would be produced in the vicinity of the surface during the ablation phase. This is possible if a laser supported plasma is formed in the plume of gas products, or at the surface of the polymer, like in the case of other materials ${ }^{34}$ at comparable fluence. An intense luminescence of longer wavelength may then penetrate deeper in the bulk.

\section{MECHANISM AND THERMAL EFFECTS}

The energy diagram of Figure 9 displays some of the elementary processes following the absorption of a far-UV photon. Upon absorption of a far-UV photon(1), a direct decomposition (5) into primary products is possible if the excited moiety is located at the surface of the material and if one of the products is small enough to be volatile. If the chromophore is embedded in the polymer subsurface, part of the energy of the absorbed photon is dissipated very quickly (a few picoseconds) into heat by the vibrational relaxation (2), or leads to a bond breaking by pathway (4) or (6). If the radical pair formed is sufficiently "free," which is the case of polymers with large free volume or melted polymer, it can live longer and avoid recombinaison to give degradation products (7). If it has no freedom, like in solid polymers, recombination (8) is the most probable event, and thermal energy is generated. Thermal energy is then principally produced by vibrational relaxation (2) and (3) recombinaison of broken bonds (8). However from these sources, (2) is the quickest since the excited state $S_{1}$ and biradicals can store electronic potential energy during their lifetimes that are of the order of nanoseconds or tens of nanoseconds. Absorption of a second photon (9) leads to a similar behaviour.

This diagram leads to a complicated kinetic scheme which is still far from being completely understood. We can say that, as a general trend, a low intensity

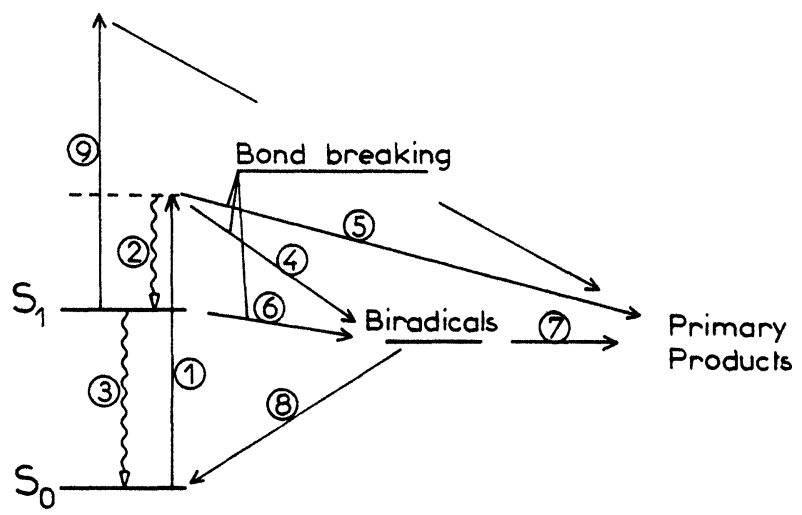

Figure 9 Energy diagram of the elementary events following the asorption of 1 or 2 far-UV photon. (1) One photon absorption (fs); (2) Vibrational relaxation to the first excited state level (ps); (3) Internal conversion (ns); (4), (5), (6) Dissociation or bond breaking (ps or ns); (7) Products formation; (8) Cage recombination; (9) Second photon absorption which can be followed by the same set of events. 
irradiation favours a quantitative thermal coupling, whereas a high intensity photolysis supports an efficient degradation which is necessarily accompanied by a proportional, but small, heat production in the solid. Despite this thermal coupling, ablation without melting of the surface of PET was seen at the low fluence of the 193 nm radiation..$^{22,42}$ Higher fluence leads to a clearly visible melting of the surface. The so called thermal loading has been measured by several authors. ${ }^{43}$ This scheme is valid for far-UV photons whose energy is much larger than that of the first excited state. A radiation of longer wavelength would require the stepwise absorption of two or more photons, which has for main consequence to permit a more extensive vibrational relaxation at each step giving more thermal effect. Delivering the far-UV photons in a shorter pulse should not basically change the scheme although the dynamics leading to ablation may be altered. In this particular case the two photon absorption, taking place preferentially at the surface, may help significantly the ablation.

\section{PERFORMANCES OF POLYMER FAR-UV LASER ABLATION}

Many researchers are interested in laser ablation as an etch tool. The photoablative behaviour of a given polymer can be characterized by the rate of ablation, experimentally given by the etch curves like those of Figures 4 and 5 . The lateral resolution of the etching is submicronic and it is not limited by the thermal effects of the ablation step. High aspect ratio can be readily achieved. Figure 10 shows scanning electron micrographs of various polymers (PET, PS, PI) that were etched by many pulses at $193 \mathrm{~nm}$, through a $400 \mu \mathrm{m}$ pinhole. In all cases, the etch depth can be very well controlled by adjusting either one or all of the following parameters; fluence, repetition rate, dose. The interface moves evenly over the irradiated area, by a discrete amount at each pulse.

Some polymer like PET develops a curious granular structure upon ablation. This phenomenon, which is due to the presence of crystallinity in the polymer film, offers a new type of rough surface having interesting properties. ${ }^{44}$ Similarly, inhomogeneous polymers or materials containing impurities or small particles are etched unevenly. In some cases the formation of conical structures at the surface was observed. ${ }^{45}$

\section{CONCLUSIONS}

Photoablation of polymers with the high intensity far-UV radiation of an excimer laser is a spontaneous etching of the irradiated surface, which results from the fast degradation (within picoseconds) of the collectively excited chains into volatile fragments. Ablation occurs only when the absorbed intensity surpasses a threshold which is characteristic of the polymer. The experimental data are supported by a dynamic model (picosecond timescale), which considers that the surface being ablated moves at a speed which is proportional to the fraction over the threshold, of 

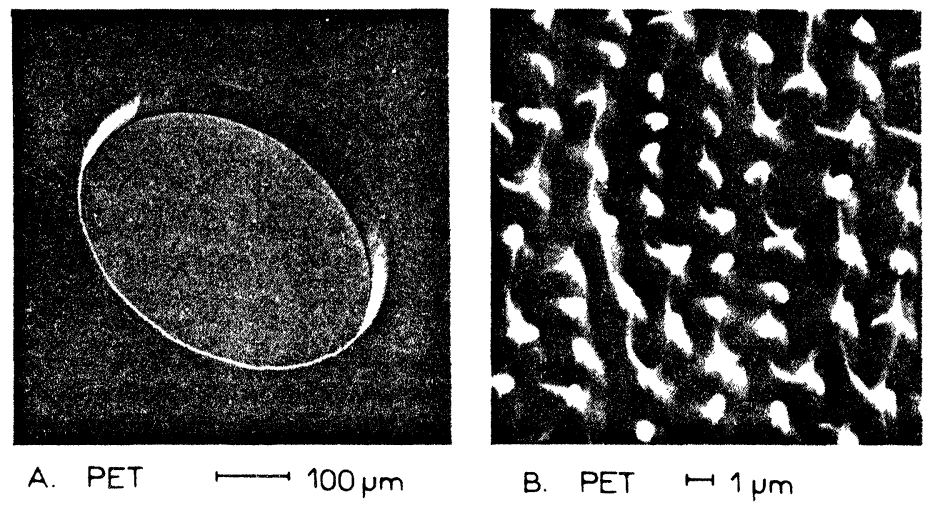

$$
\text { B. PET } \mapsto 1 \mu \mathrm{m}
$$
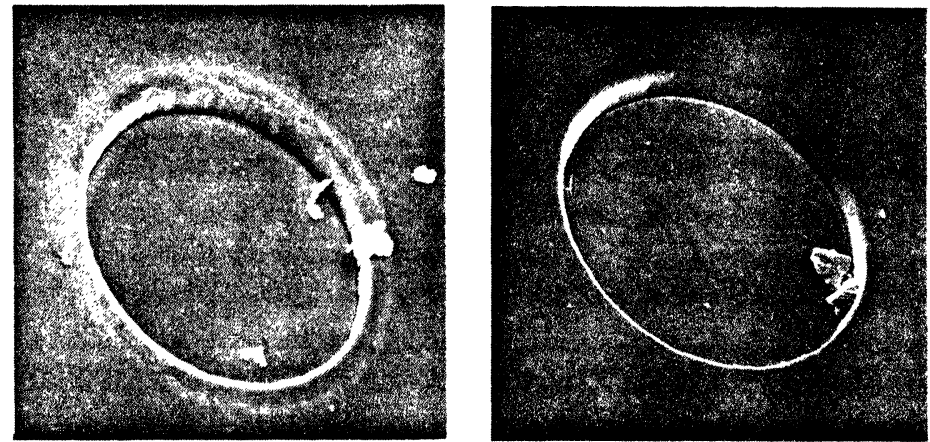

C. PS

$100 \mu \mathrm{m}$

D. PI $\mapsto 1 \mu \mathrm{m}$

Figure 10 Scanning electron micrographs of polymer surfaces etched with many pulses of the $193 \mathrm{~nm}$ radiation of the excimer laser $\left(200 \mathrm{~mJ} / \mathrm{cm}^{2}\right)$ through a $400 \mu \mathrm{m}$ pinhole mask. (a) poly(ethylene terephthalate); (b) high magnification of the PET surface showing the roughening obtained by ablation; (c) polystyrene; (d) polyimide. Note that the surface of these two polymers remains smooth after etching by photoablation.

the intensity effectively falling on the moving interface $v=k\left(I_{0}(t) e^{-\beta x(t)}-I_{t}\right)$ (taking into account the reabsorption by the gas products). By integrating the rate law we can synthesize the etch curves, accurately measured with the microbalance. Two parameters $\beta$ and $k$ can be extracted for a given couple of polymer and wavelength. From this model, was computed the deposited energy as a function of depth under the starting surface. A considerable part of the incident pulse is reabsorbed by the gas products.

\section{Acknowledgements}

The authors thank Professor Joussot Dubien for his interest in this work and Bernard Sillon for a gift of polyphenylquinoxaline.

\section{References}

1. Time elapsed since Srinivasan's first paper on ablative photodecomposition: R. Srinivasan and V. Mayne-Banton, Appl. Phys. Lett. 41, 576 (1982). 
2. D. Bastings and B. Nikolaus, Springer Proceedings in Physics 15, 410 (1987).

G. Klauminzer, Lasers and Applications 73 (1986).

T. A. Znotins, D. Poulin and J. Reid, Laser Focus/Electro-Optics 54 (1987).

3. C. A. Brau, Topics in Appl. Phys. 30, 87 (1979).

4. R. Srinivasan, Science 234, 559 (1986).

5. K. J. Ilicisin and R. Fedosejevs, Appl. Opt. 26, 396 (1987).

6. T. A. Znotins, Lasers and Appl. 5, 71 (May 86). and Laser Focus/Electro-Optics, 54 (May 87).

G. T. Forest, Laser Focus/Electro-Optics 28 (May 87).

Y. S. Liu, H. S. Cole, H. R. Philipp and R. Guida, Proc. SPIE-Int. Soc. Opt. Eng. 774, 155 (1987).

D. J. Elliott, K. J. Polasko, B. P. Piwczyk and E. W. Balch, SPIE-Int. Soc. Opt. Eng. 774, 172 (1987).

S. E. Blum, K. Brown and R. Srinivasan, US Patent \#4414059 (1983).

J. H. Brannon and J. R. Lankard, US Patent \#4508749 (1985).

S. E. Blum, K. L. Holloway and R. Srinivasan, US Patent \#4568632 (1986).

H. S. Cole, Y. S. Liu and H. R. Philipp, US Patent \#4617085 (1986).

J. J. Donelon, Y. Tomkiewicz, T. A. Wassick and J. T. Yeh, Eur. Pat. Appl. \#0227903 (1986).

7. D. Muller, Laser and Appl. 5, 86 (May 86).

N. P. Furzikov, IEEE J. Quant. Elect. QE-23, 1751 (1987).

8. R. Iscoff, Lasers and Optronics 6, 65 (Nov. 1987).

C. A. Puliafito and R. F. Steinert, Laser Focus/Electro-Optics 85 (Sept. 1985).

C. A. Puliafito, R. F. Steinert, T. F. Deutsch, F. Hillenkamp, E. J. Dehm and C. M. Adler, Ophthalmology 92, 741 (1985).

J. A. Marshall, S. Trokel, S. Rothery and R. R. Krueger, Brit. J. Ophth. 70, 482 (1986).

9. Ref. 6 and J. M. Isner, P. G. Steg and R. H. Clarke, IEEE J. Quant. Electr. QE-23, 1756 (1987).

10. H. Nornes, R. Srinivasan, R. Solanski and E. Johnson, Soc. Neurosci. 11, 1167 (1985).

11. See Ref. 6.

12. For instance tungsten, palladium and new metal oxide superconductors, D. Dijkkamp, T. Venkatesan, X. D. Wu, S. A. Shaheen, N. Jisrawi, Y. H. Min-Lee, W. L. McLean and M. Croft, Appl. Phys. Lett. 51, 619 (1987).

13. F. Hillenkamp, E. Unsold, R. Kaufmann and R. Nitsche, Appl. Phys. 8, 341 (1975).

R. Holm, M. Karas and H. Vogt, Anal. Chem. 0359, 371 (1987).

14. I. Hussla and R. Viswanathan, Surface Sci. Lett. 145, 488 (1984).

15. G. B. Shinn, F. Steigerwald, H. Stiegler, R. Sauerbrey, F. K. Tittel and W. L. Wilson, J. Vac. Sci. Technol. B4, 1273 (1986).

16. A. Inam, X. D. Wu, T. Venkatesan, S. B. Ogale, C. C. Chang and D. Dijkkamp, Appl. Phys. Lett. 51, 1112 (1987).

17. R. Srinivasan, J. Vac. Sci. Technol. B1, 923 (1983).

18. Cornea, arthery, skin and nerve tissue have already been studied see Ref. 7.

19. P. E. Dyer, R. Srinivasan and B. Braren, Lasers Surg. Med. (in press).

20. Only a small part of the incident energy hits the solid target, the excess of energy is carried away with the ablated products.

21. S. Lazare, P. D. Hoh, J. M. Baker and R. Srinivasan, J. Amer. Chem. Soc. 106, 4288 (1984); R. Srinivasan and S. Lazare, Polymer 26, 1287 (1985) and S. Lazare and R. Srinivasan, J. Phys. Chem. 90, 2124 (1986).

22. S. Lazare and V. Granier, J. Appl. Phys. 63, 2110 (1988).

23. B. J. Garrison and R. Srinivasan, Appl. Phys. Lett. 44, 849 (1984); J. Appl. Phys. 57, 2909 (1985).

24. P. E. Dyer and R. Srinivasan, Appl. Phys. Lett. 48, 445 (1986); R. Srinivasan, P. E. Dyer and B.

Braren, Lasers Surg. Med. (in press).

25. T. Keyes, R. H. Clarke and J. M. Isner, J. Phys. Chem. 89, 4194 (1985).

26. E. Sutcliffe and R. Srinivasan, J. Appl. Phys. 60, 3315 (1986).

27. G. Koren, Appl. Phys. Lett. 50, 1030 (1987).

28. G. M. Davis and M. C. Gower, J. Appl. Phys. 61, 2090 (1987).

29. J. Meyer, J. Kutzner, D. Feldmann and K. H. Welge, Appl. Phys. B45, 7 (1988).

30. S. Lazare, J.C. Soulignac and P. Fragnaud, Appl. Phys. Lett. 50, 624 (1987).

31. R. Srinivasan, E. Sutcliffe and B. Braren, Appl. Phys. Lett. 51, 1285 (1987).

32. R. Srinivasan and B. Braren, J. Polym. Sci. Polym. Chem. Edit. 22, 2601 (1984).

B. Braren and D. Seeger, J. Polym. Sci. Polym. Lett. Edit. 24, 371 (1986).

33. J. E. Andrew, P. E. Dyer, D. Forster and P. H. Key, Appl. Phys. Lett. 43, 717 (1983).

34. R. Poprawe, E. Beyer and G. Herziger, Inst. Phys. Conf. Ser. 72,67 (1984).

G. Herziger and E. W. Krentz, Spring. Ser. Chem. Phys. 39, 90 (1984).

35. R. Srinivasan and W. J. Leigh, J. Amer. Chem. Soc. 104, 6784 (1982). 
J. H. Brannon, J. R. Lankard, A. I. Baise, F. Burns and J. Kaufman, J. Appl. Phys. 58, 2036 (1985). 36. G. Koren and J. T. C. Yeh, Appl. Phys. Lett. 44, 1112 (1984).

G. Koren and J. T. C. Yeh, J. Appl. Phys. 56, 2120 (1984).

G. M. Davis, M. C. Gower, C. Fotakis, T. Efthimiopoulos and P. Argyrakis, Appl. Phys. A36, 27 (1985).

37. R. Srinivasan, B. Braren, D. E. Seeger and R. W. Dreyfus, Macromolecules 19, 916 (1986) and R.

Srinivasan, B. Braren, R. W. Dreyfus, L. Hadel and D. E. Seeger, J. Opt. Soc. Am. B/3, 785 (1986).

R. Srinivasan, B. Braren and R. W. Dreyfus, J. Appl. Phys. 61, 372 (1986).

38. B. Danielzk, N. Fabricius, M. Röwekamp and D. von der Linde, Appl. Phys. Lett. 48, 212 (1986).

R. C. Estler and N. S. Nogar, Appl. Phys. Lett. 49, 1175 (1986).

R. Larciprete and M. Stuke, Appl. Phys. B42, 181 (1987).

D. Feldmann, J. Kutzner, J. Laukemper, S. MacRobert and K. H. Welge, Appl. Phys. B44, 81 (1987).

39. D. L. Singleton, G. Paraskevopoulos, G. S. Jolly, R. S. Irwin, D. J. McKenney, W. S. Nip, E. M. Farrell and L. A. J. Higginson, Appl. Phys. Lett. 48, 878 (1986).

D. L. Singelton, G. Paraskevopoulos, R. S. Taylor and L. A. J. Higginson, IEE J. Quant. Electron. QE-23, 1772 (1987).

J. M. Isner, P. G. Steg and R. H. Clarke, IEEE J. Quant. Electron. QE-23, 1756 (1987).

40. R. J. von Gutfeld and R. Srinivasan, Appl. Phys. Lett. 51, 15 (1987).

41. R. E. Walkup, J. M. Jasinski and R. W. Dreyfus, Appl. Phys. Lett. 48, 1690 (1986).

42. Y. Novis, J. J. Pireaux, A. Brezini, E. Petit, R. Caudano, P. Lutgen, G. Feyder and S. Lazare, J. Appl. Phys. 64, 365 (1988).

43. G. Gorodetsky, T. G. Kazyaka, R. L. Melcher and R. Srinivasan, Appl. Phys. Lett. 46, 828 (1985).

R. W. Dreyfus, F. A. McDonald and R.J. von Gutfeld, J. Vac. Sci. Technol. B5, 1521 (1987).

44. S. Lazare, V. Granier, P. Lutgen and G. Feyder, Rev. Phys. Appl. 23, 1065 (1988).

45. P. E. Dyer, S. D. Jenkins and J. Sidhu, Appl. Phys. Lett. 49, 453 (1986). 